\title{
A low-rank algorithm for weakly compressible flow ${ }^{\text {wh }}$
}

\author{
Lukas Einkemmer ${ }^{\mathrm{a}, \mathrm{b}, *}$ \\ ${ }^{a}$ Department of Mathematics, University of Tübingen, Germany \\ ${ }^{b}$ Department of Mathematics, University of Innsbruck, Austria
}

\begin{abstract}
In this paper, we propose a numerical method for solving weakly compressible fluid flow based on a dynamical low-rank projector splitting. The low-rank splitting scheme is applied to the Boltzmann equation with BGK collision term, which results in a set of constant coefficient advection equations. This procedure is numerically efficient as a small rank is sufficient to obtain the relevant dynamics (described by the Navier-Stokes equations). The resulting method can be combined with a range of different discretization strategies; in particular, it is possible to implement spectral and semi-Lagrangian methods, which allows us to design numerical schemes that are not encumbered by the sonic CFL condition.
\end{abstract}

Keywords: dynamical low-rank approximation, projector splitting, Boltzmann equation, fluid dynamics, weakly compressible flow

\section{Introduction}

Fluids play a pivotal role in virtually all fields of science and engineering. Consequently, computational fluid dynamics (CFD) is used from modeling pipe flows on a single workstation to simulating airplanes or turbulent combustion on state of the art supercomputers. The governing partial differential equations (PDEs) are the Navier-Stokes equations. More specifically, in the present work we will consider the compressible isothermal Navier-Stokes equations

$$
\begin{aligned}
\partial_{t} \rho+\nabla_{x}(\rho u) & =0, \\
\partial_{t}(\rho u)+\nabla \cdot(\rho u \otimes u)+\nabla p & =\nabla \cdot\left[\mu\left(\nabla u+(\nabla u)^{\mathrm{T}}\right)+\lambda(\nabla \cdot u) I\right] \\
p & =\rho \theta,
\end{aligned}
$$

where the density $\rho$ and the momentum $\rho u$ are the sought-after quantities. Since we consider the isothermal case the (thermodynamic) temperature $\theta$ is fixed. The pressure is determined by the ideal gas law $p=\rho \theta$. Two material

\footnotetext{
* Corresponding author

Email address: lukas.einkemmer@uibk.ac.at (Lukas Einkemmer)
} 
parameters, the dynamic viscosity $\mu$ and the volume viscosity $\lambda$ have to be specified. In the case of vanishing viscosity (i.e. $\mu=0$ and $\lambda=0$ ) equations (1) are usually referred to as the Euler equations.

The most common approach to solving these equations numerically is to discretize them on an appropriate grid. Historically finite difference and finite volume methods have been used extensively, while in recent years discontinuous Galerkin schemes have become more common. However, especially in the study of turbulence by direct numerical simulation (DNS), spectral methods are often preferred (see, for example, [13, 34]).

This approach (which we will refer to as direct discretization in the following) is very mature and sophisticated numerical methods have been developed in the last decades. Further advantages of this approach are that (at least the basic) numerical algorithms are often easy to understand and implement. Disadvantages include that explicit methods usually need to satisfy the CFL condition for sound waves (which in the weakly compressible setting can be multiple orders of magnitude faster than the speed of flow) and that the equations are relatively complicated (which puts significant constraints on the design of numerical methods).

However, a direct discretization of the Navier-Stokes equations is not the only way to perform fluid simulations. In particular, lattice Boltzmann, methods have been considered extensively in the literature (see, for example, [5, 18, 17]). The lattice Boltzmann method exploits the fact the Boltzmann equation (a kinetic model), for an appropriately modeled collision term and initial value, recovers the dynamics of the Navier-Stokes equations (see, for example, 3, 2]). Thus, in principle, we can solve fluid flow problems by integrating the Boltzmann equation in time. However, these kinetic problems are posed in a $2 d$ dimensional phase space ( $d$ dimensions of space, as for the Navier-Stokes equations, and $d$ dimensions of velocity). Thus, a direct discretization is prohibitively expensive from a computational point of view. The sought-after quantity, a distribution function or particle-density, is usually denoted by $f(t, x, v)$.

However, in the fluid regime (i.e. for thermalized gases or liquids) we know that the distribution in velocity space stays close to a Maxwell-Boltzmann distribution. That is,

$$
f(t, x, v) \approx \frac{\rho(t, x)}{(2 \pi \theta)^{d / 2}} \exp \left(-\frac{1}{2}(v-u(t, x))^{2}\right) .
$$

What we actually want to approximate are the moments of $f$ (which correspond to the macroscopic quantities of density $\rho$ and momentum $\rho u$ ). These are quantities of interest in fluid simulations (as opposed to the distribution function $f$ ). The idea of the lattice Boltzmann method is to discretize the velocity space with only a small number of discrete velocities $e_{j} \in \mathbb{R}^{d}$. Then, the moments can be computed using a Gaussian-type quadrature 


$$
\begin{aligned}
\rho(t, x) & =\int f(t, x, v) \mathrm{d} v \approx \sum_{j} W_{j} f_{j}(t, x), \\
\rho u(t, x) & =\int v f(t, x, v) \mathrm{d} v \approx \sum_{j} W_{j} e_{j} f_{j}(t, x),
\end{aligned}
$$

where $f_{j}(t, x) \approx f_{j}\left(t, x, e_{j}\right)$ and $W_{j}$ are quadrature weights. We then only have to solve an evolution equation for the (relatively) small number of $f_{j} \mathrm{~s}$ (which are $d$-dimensional functions of $x$ ). For the classic lattice Boltzmann method in two-dimensions, the $e_{j}$ are chosen as the corners of a square and the zero vector. Thus, we have $9 f_{j}$ in two-dimensions (this is referred to as D2Q9). In three dimensions a variety of schemes have been considered (for example, D3Q19 and D3Q27 with 19 and $27 f_{j}$ s, respectively). If the length of the square/cube is $2 h / \tau$, where $h$ is the grid spacing and $\tau$ is the time step size, a numerical method (operated with unit CFL number) can be implemented without discretizing any differential operators. This is a consequence of the fact that the Boltzmann equation is much simpler compared to the Navier-Stokes equations. A further advantage of the lattice Boltzmann method is that it can usually be parallelized very efficiently. Disadvantages include that the amount of memory needed is increased (compared to a direct discretization of the Navier-Stokes equations) and that the method is most effective if simulations are conducted using a unit CFL number. Attempts to overcome the latter limitation have resulted in the developed of so-called off-lattice Boltzmann methods (see, for example, [29, 9, 17]). However, according to [17] these methods can be computationally expensive due to the high number of partial differential equations that have to be solved.

In the present paper we propose an alternative approach to both a direct discretization of the Navier-Stokes equations and to the lattice Boltzmann method. Similar to the lattice Boltzmann method our scheme is applied to the Boltzmann equation. However, to reduce the dimensionality of the problem (from $2 d$ to $d$ ) we perform a low-rank approximation. We then obtain evolution equations that describe the dynamics of the Boltzmann equation constraint to the corresponding low-rank manifold. To accomplish this the dynamical low-rank splitting algorithm introduced in 22] is used. This allows us to represent the evolution in velocity space in more detail. In fact, we obtain evolution equations for functions that depend on $x$, but not on $v$ (as in the lattice Boltzmann method). However, we also obtain similar evolution equations for functions that depend only on $v$, but not on $x$.

In addition, the evolution equations obtained are still significantly simpler compared to the Navier-Stokes equations (essentially we obtain a constantcoefficient advection with an inhomogeneity). Thus, a range of space discretization strategies can be employed relatively easily. In particular, we can use (true) spectral methods (as opposed to the pseudo-spectral approach which is common for the direct discretization of the Navier-Stokes equations). Furthermore, it is possible within this approach to construct a numerical method that can over- 
come the CFL condition imposed by the speed of sound. This is particular relevant for weakly compressible simulations. Another interesting property of the projector-splitting integrator is that it mimics the properties of the (continuous) Boltzmann equation as we approach the limit of vanishing viscosity (i.e. as we consider the limit that yields the Euler equations from the Navier-Stokes equations).

Let us note that low-rank approximations have been extensively used in quantum mechanics. See, in particular, [28, 27] for the MCTDH approach to molecular quantum dynamics in the chemical physics literature and [20, 21, 6] for a computational mathematics point of view. Some uses of dynamical lowrank approximation in areas outside quantum mechanics are described in [31, 11, 26, 30]. In a general mathematical setting, dynamical low-rank approximation has been studied in 14, 15, 24. A major algorithmic advance for the time integration was achieved with the projector-splitting methods first proposed in 22 for matrix differential equations and then developed further for various tensor formats in [21, 23, 10, 12, 25]. Low-rank approximations for computational plasma physics (i.e. the collisionless but magnetized Boltzmann equation) have been considered in [16, 7]. Note, however, these schemes try to capture kinetic effects that occur far away from thermodynamic equilibrium. This means that the Navier-Stokes equations (or any other model that considers only the moments of $f$ ) are not applicable in this setting.

The outline of this paper is as follows. First, we summarize how the Boltzmann equation gives rise to the Navier-Stokes equations (section 2). Then we introduce the proposed numerical algorithm (section 3). In section 4 we investigate the behavior of the low-rank projector-splitting as the viscosity vanishes. Numerical results are presented in section 5. Finally, we conclude in section 6.

\section{Obtaining fluid dynamics from the Boltzmann equation}

The compressible isothermal Navier-Stokes equations have already been stated in (1). The goal in this section is to show how the dynamics of the Navier-Stokes equations arises from the Boltzmann equation. Although, this has been investigated before [3, 2, it is essential to motivate and explain the numerical algorithm that is described in section 3 .

In the remainder of the paper we will non-dimensionalize the Navier-Stokes equations as follows. First, we choose a characteristic length scale $L$ (in section 5 this will be the length of the computational domain). Then we choose the speed of sound $c_{s}$ as the characteristic velocity. Since the speed of sound is

given by $c_{s}=\sqrt{\frac{\partial p}{\partial \rho}}=\sqrt{\theta}$ this choice implicitly sets $\theta=1$. As a consequence the characteristic time is then $T=L / c_{s}$. This is just the time it takes a sound wave to propagate from one end of the domain to the other end.

Since we focus on weakly compressible flow here, the flow speed is always appreciably smaller than 1 (the speed of sound) and we initialize our problem with a homogeneous fluid density. The Navier-Stokes equations remain invariant with respect to scaling the density (i.e. only variations in the density are 
important). Thus, we simply initialize $\rho=1$. For a real fluid this would determine the units used to measure mass and thus also (uniquely) determines the units used to measure viscosity. A flow with speed $u$ then has a Reynolds number (in non-dimensionalized units)

$$
\operatorname{Re}=\frac{\rho u L}{\mu}=\frac{u}{\mu}
$$

and a Mach number

$$
\mathrm{M}=\frac{u}{c_{s}}=u .
$$

It is also instructive to discuss the incompressible limit. In this case the (now incompressible) Navier-Stokes equations become

$$
\begin{aligned}
\partial_{t}(\rho u)+\nabla \cdot(\rho u \otimes u)+\nabla p & =\mu \Delta u, \\
\nabla \cdot u & =0 .
\end{aligned}
$$

Formally, this is obtained by taking $c_{s} \rightarrow \infty$. This also explains the infinite speed of propagation modeled by the divergence free constraint $\nabla \cdot u=0$. Clearly it is then not possible to use the speed of sound as a characteristic velocity. Instead a typical velocity of the flow is usually chosen. Care has to be taken when comparing weakly compressible simulation results (such as those in section 5) with incompressible simulations (such as those in [4, 19, 8]). In particular, in the latter the characteristic time is $T_{\text {incompr }}=L / u$ and in the former $T_{\text {compr }}=L / c_{s}=\mathrm{M} L / u$. Thus, the final time of a simulation has to be adjusted accordingly.

We now consider the Boltzmann equation

$$
\partial_{t} f_{\epsilon}(t, x, v)+v \cdot \nabla_{x} f_{\epsilon}(t, x, v)=\frac{1}{\epsilon} C\left(f_{\epsilon}\right)(x, v),
$$

where $C$ is the collision operator and $\epsilon$ is a (usually small) parameter. The sought-after quantity is the phase space distribution $f_{\epsilon}$. From a physical point of view the collision operator has to enforce that the dynamics stays close to a Maxwell--Boltzmann distribution in velocity space. Consequently, we assume that

$$
C(g)=0
$$

has solutions that can be written in the following form

$$
g(x, v)=\frac{\rho(x)}{(2 \pi \theta)^{d / 2}} \exp \left(-\frac{1}{2} \frac{(v-u(x))^{2}}{\theta}\right),
$$

where the density $\rho$ and the velocity $u$ (strictly speaking, the momentum $\rho u$ ), are given by the moments

$$
\rho(x)=\int g(x, v) \mathrm{d} v, \quad \rho u(x)=\int v g(x, v) \mathrm{d} v .
$$


From now on we set the (thermodynamic) temperature to one (i.e. $\theta=1$ ). Equation (6) is precisely what we would, on physical grounds, expect from an ideal thermalized fluid.

The remarkable observation here is that, equation (4) still fully captures the (very complicated) dynamics of the Navier-Stokes equations. Conceptually the simplest case is the limit $\epsilon \rightarrow 0$. Thus, we will consider it here. In this case the right-hand side of equation (4) constrains the solution to the form

$$
f^{\mathrm{eq}}(t, x, v)=\frac{\rho(t, x)}{(2 \pi)^{d / 2}} \exp \left(-\frac{1}{2}(v-u(t, x))^{2}\right),
$$

where $\rho$ and $u$ are, as of yet, undetermined quantities. Thus, we have $f^{\mathrm{eq}}=$ $\lim _{\epsilon \rightarrow 0} f_{\epsilon}$. We proceed by integrating equation (4) with respect to velocity and obtain

$$
\partial_{t} \int f_{\epsilon} \mathrm{d} v+\nabla_{x} \cdot\left(\int v f_{\epsilon} \mathrm{d} v\right)=\frac{1}{\epsilon} C\left(f_{\epsilon}\right)
$$

Now, we take the (formal) limit $\epsilon \rightarrow 0$

$$
\partial_{t} \int f^{\mathrm{eq}}(t, x, v) \mathrm{d} v+\nabla_{x} \cdot\left(\int v f^{\mathrm{eq}}(t, x, v) \mathrm{d} v\right)=0 .
$$

Note that the collision term has vanished as the solution $f^{\text {eq }}$ given by equation (7) satisfies (5). Employing the definition of density and velocity as the moments of the phase space density, we easily obtain

$$
\partial_{t} \rho+\nabla_{x} \cdot(\rho u)=0 .
$$

This is precisely the continuity equation.

To derive the momentum balance equations, we multiply equation (4) by $v_{j}$ (the $j$ th component of the velocity) and integrate in velocity space. This yields

$$
\partial_{t} \int v_{j} f_{\epsilon} \mathrm{d} v+\sum_{i} \partial_{x_{i}} \int v_{j} v_{i} f_{\epsilon} \mathrm{d} v=\frac{v_{j}}{\epsilon} C\left(f_{\epsilon}\right) .
$$

Taking the limit $\epsilon \rightarrow 0$ gives

$$
\partial_{t}\left(\rho u_{j}\right)+\sum_{i} \partial_{x_{i}} \int v_{j} v_{i} f^{\text {eq }} \mathrm{d} v=0 .
$$

We now evaluate the resulting integrals by using equation (7). We obtain

$$
\int v_{j} v_{i} f^{\mathrm{eq}} \mathrm{d} v= \begin{cases}\rho\left(u_{i}^{2}+1\right) & i=j \\ \rho u_{i} u_{j} & i \neq j\end{cases}
$$

and thus

$$
\partial_{t}(\rho u)+\nabla \cdot(\rho u \otimes u)+\nabla \rho=0 .
$$

This is precisely the momentum balance equation for an ideal gas. More canonically we would write this using the pressure $p$ and impose the equation of state 
corresponding to an ideal gas, i.e. $p=\rho$. In summary, we have obtained the compressible isothermal Euler equations.

The question that remains to be answered is why a low-rank representation makes sense here. We know that the solution satisfies the form specified by equation (7) at all times. However, this is not a low-rank representation due to the presence of both velocity $(v)$ and position $(x)$ dependent functions in the exponential. However, if the flow velocity is small compared to the speed of sound (i.e. in the weakly compressible case) we can use

$$
f^{\mathrm{eq}}=\frac{\rho}{(2 \pi)^{d / 2}} \exp \left(-\frac{v^{2}}{2}\right)\left(1+v \cdot u+\frac{(v \cdot u)^{2}}{2}-\frac{u^{2}}{2}\right)+\mathcal{O}\left(u^{3}\right) .
$$

This is a low-rank approximation with rank 6 and 10 for two- and threedimensional problems, respectively. For comparison, a lattice Boltzmann method usually requires 9 directions in two-dimensions and 19 to 27 directions in threedimensions (see the discussion in the introduction). Thus, at least in princple, representing the solution by a low-rank repersentation is a viable approach.

The derivation for the Navier-Stokes equations (i.e. for $\epsilon>0$ ) is more involved. One proceeds by performing a Chapman-Enskog expansion. That is, we assume that $\epsilon$ is a small parameter and look for a solution, up to terms of $\mathcal{O}\left(\epsilon^{2}\right)$, to equation 4 that has the form

$$
f_{\epsilon}=f^{\mathrm{eq}}\left(1+\epsilon g_{\epsilon}+\epsilon^{2} w_{\epsilon}\right),
$$

where $f^{\text {eq }}$ is given, as before, in the form specified by equation (7). The functions $g_{\epsilon}$ and $w_{\epsilon}$ give, respectively, the first and second order deviation from $f^{\text {eq }}$ caused by the finite $\epsilon$. We will use the BGK (Bhatnagar-Gross-Krook) collision operator

$$
C\left(f_{\epsilon}\right)=f^{\mathrm{eq}}-f_{\epsilon} .
$$

It is easy to check that this collision operator satisfies the condition given in (5). The BGK collision operator is heavily used in lattice Boltzmann simulations and we will also employ it for the numerical results conducted in section 5 .

It can then be shown that we recover the continuity equation

$$
\partial_{t} \rho+\nabla \cdot(\rho u)=0
$$

and the following momentum balance equations

$$
\partial_{t}(\rho u)+\nabla \cdot(\rho u \otimes u)+\nabla \rho=\epsilon \nabla \cdot\left[\rho \nabla u+\rho(\nabla u)^{\mathrm{T}}-\frac{2}{d} \rho(\nabla \cdot u) I\right] .
$$

For more details of the derivation we refer the reader to [3, 2, 33. Comparing this to the Navier-Stokes equations stated in the introduction, i.e. equation (1), we have a perfect match, except for the diffusion term. However, in the case of weakly compressible flow $\rho$ varies only slightly. In addition, nondimensionalization allows us to set the characteristic value of $\rho$ to unity. Thus, making the approximation $\rho \approx 1$ and applying it to the right-hand side of equation 10 we obtain

$$
\partial_{t}(\rho u)+\nabla \cdot(\rho u \otimes u)+\nabla \rho=\epsilon \nabla \cdot\left[\nabla u+(\nabla u)^{\mathrm{T}}-\frac{2}{d}(\nabla \cdot u) I\right] .
$$


Thus, we have recovered the Navier-Stokes equation with $\mu=\epsilon$ and $\lambda=-2 \epsilon / d$.

It should also be noted that, while there is no guarantee, that $g_{\epsilon}$ is a low-rank function, the dynamics implied by the Navier-Stokes equations forces $f_{\epsilon}$ to stay close to a low-rank function. This further motivates the proposed approach and we will see in section 5 that usually quite low ranks are sufficient in order to obtain excellent agreement with the dynamics of interest.

\section{Numerical method}

We start from the Boltzmann equation

$$
\partial_{t} f(t, x, v)+v \cdot \nabla_{x} f(t, x, v)=\frac{1}{\epsilon} C(f)(x, v),
$$

with the BGK collision operator

$$
C(f)=f^{\text {eq }}-f,
$$

where $\epsilon>0$ is a (usually small) parameter and

$$
f^{\mathrm{eq}}(t, x, v)=\frac{\rho(t, x)}{(2 \pi)^{d / 2}} \exp \left(-\frac{1}{2}(v-u(t, x))^{2}\right),
$$

where $d \in\{1,2,3\}$ is the dimension of the problem. The sought-after quantity is $f$ (in this section we will not explicitly denote the dependence of $f$ on $\epsilon$ ). As has been outlined in the previous section, the moments

$$
\rho=\int f \mathrm{~d} v, \quad \rho u=\int v f \mathrm{~d} v
$$

then satisfy the compressible isothermal Navier-Stokes equations. As initial value we choose a function of the form

$$
f(0, x, v)=\frac{\rho^{0}(x)}{(2 \pi)^{d / 2}} \exp \left(-\frac{1}{2}\left(v-u^{0}(x)\right)^{2}\right),
$$

This is not yet a low-rank representation. However, in an actual implementation we can either use the expansion given in equation (8) or perform a singular value decomposition (SVD) once the problem is discretized.

What remains to be determined here is the density $\rho^{0}$ and the velocity $u^{0}$ (or alternatively, the momentum $\rho u^{0}$ ). These are specified according to the fluid problem for which a numerical solution is sought.

Since equation (11) is posed in a $2 d$ dimensional phase space, its direct solution is prohibitively expensive. This is particularly true in the present setting as the dynamics stays close to a low-rank manifold (see the discussion in the previous section). Thus, the goal of this section is to derive an algorithm that approximates the Boltzmann equation (11) by a low-rank representation. 
To that end, the function $f(t, x, v)$ is constrained to the following form

$$
f(t, x, v)=\sum_{i j} X_{i}(t, x) S_{i j}(t) V_{j}(t, v)
$$

where $S \in \mathbb{R}^{r \times r}$ and we call $r$ the rank of the representation. Note that the dependence of $f$ on the phase space $(x, v) \in \Omega \subset \mathbb{R}^{2 d}$ is now approximated by the functions $\left\{X_{i}: i=1, \ldots, r\right\}$ and $\left\{V_{j}: j=1, \ldots, r\right\}$ which depend only on $x \in \Omega_{x} \subset \mathbb{R}^{d}$ and $v \in \Omega_{v} \subset \mathbb{R}^{d}$, respectively. In equation 12 and the following discussion we always assume that summation indices run from 1 to $r$ and we thus do not specify these bounds.

Now, we seek an approximation to the exact particle-density function that for all $t$ lies in the set

$\overline{\mathcal{M}}=\left\{f \in L^{2}(\Omega): f(x, v)=\sum_{i j} X_{i}(x) S_{i j} V_{j}(v)\right.$ with $\left.S \in \mathbb{R}^{r \times r}, X_{i} \in L^{2}\left(\Omega_{x}\right), V_{j} \in L^{2}\left(\Omega_{v}\right)\right\}$.

It is clear that this representation is not unique. In particular, we can make the assumption that $\left(X_{i}, X_{k}\right)=\delta_{i k}$ and $\left(V_{j}, V_{l}\right)=\delta_{j l}$, where $(\cdot, \cdot)$ is the inner product on $L^{2}\left(\Omega_{x}\right)$ and $L^{2}\left(\Omega_{v}\right)$, respectively. We consider a path $f(t)$ on $\overline{\mathcal{M}}$. The corresponding derivative is denoted by $\dot{f}$ and is of the form

$$
\dot{f}=\sum_{i j}\left(X_{i} \dot{S}_{i j} V_{j}+\dot{X}_{i} S_{i j} V_{j}+X_{i} S_{i j} \dot{V}_{j}\right)
$$

If we impose the conditions $\left(X_{i}, \dot{X}_{j}\right)=\left(V_{i}, \dot{V}_{j}\right)=0$ then $S_{i j}$ is uniquely determined by $\dot{f}$. This follows easily from the fact that

$$
\dot{S}_{i j}=\left(X_{i} V_{j}, \dot{f}\right) \text {. }
$$

We then project both sides of equation 13 onto $X_{i}$ and $V_{j}$, respectively, and obtain

$$
\begin{aligned}
\sum_{j} S_{i j} \dot{V}_{j} & =\left(X_{i}, \dot{f}\right)-\sum_{j} \dot{S}_{i j} V_{j}, \\
\sum_{i} S_{i j} \dot{X}_{i} & =\left(V_{j}, \dot{f}\right)-\sum_{i} X_{i} \dot{S}_{i j} .
\end{aligned}
$$

From these relation it follows that the $X_{i}$ and $V_{j}$ are uniquely defined if $S$ has full rank (this, in particular, implies that $S$ and $S^{T}$ are invertible). Thus, we seek an approximation that for each time $t$ lies in the manifold

$$
\begin{gathered}
\mathcal{M}=\left\{f \in L^{2}(\Omega): f(x, v)=\sum_{i j} X_{i}(x) S_{i j} V_{j}(v) \text { with } S \in \mathbb{R}^{r \times r}, X_{i} \in L^{2}\left(\Omega_{x}\right), V_{j} \in L^{2}\left(\Omega_{v}\right)\right. \text { and } \\
\left.\left(X_{i}, X_{k}\right)=\delta_{i k},\left(V_{j}, V_{l}\right)=\delta_{j l}, S \text { has full rank }\right\}
\end{gathered}
$$


with the corresponding tangent space

$$
\begin{array}{r}
\mathcal{T}_{f} \mathcal{M}=\left\{\dot{f} \in L^{2}(\Omega): \dot{f}(x, v)=\sum_{i j}\left(X_{i}(x) \dot{S}_{i j} V_{j}(v)+\dot{X}_{i}(x) S_{i j} V_{j}(v)+X_{i}(x) S_{i j} \dot{V}_{j}(v)\right),\right. \\
\text { with } \left.\dot{S} \in \mathbb{R}^{r \times r}, \dot{X}_{i} \in L^{2}\left(\Omega_{x}\right), \dot{V}_{j} \in L^{2}\left(\Omega_{v}\right), \text { and }\left(X_{i}, \dot{X}_{j}\right)=\left(V_{i}, \dot{V}_{j}\right)=0\right\},
\end{array}
$$

where $f$ is given by equation 12 . Now, we consider the dynamics of the Boltzmann equation on the manifold $\mathcal{M}$. That is, we consider

$$
\partial_{t} f=-P(f)\left(v \cdot \nabla_{x} f-\frac{1}{\epsilon} C(f)\right),
$$

where $P(f)$ is the orthogonal projector onto the tangent space $\mathcal{T}_{f} \mathcal{M}$, as defined above.

We will consider the projection $P(f) g$ for a moment. From equations (13)(16) we obtain

$$
P(f) g=\sum_{j}\left(V_{j}, g\right) V_{j}-\sum_{i j} X_{i}\left(X_{i} V_{j}, g\right) V_{j}+\sum_{i}\left(X_{i}, g\right) X_{i} .
$$

Let us introduce the following two vector spaces $\bar{X}=\operatorname{span}\left\{X_{i}: i=1, \ldots, r\right\}$ and $\bar{V}=\operatorname{span}\left\{V_{j}: j=1, \ldots r\right\}$. Then we can write the projector as follows

$$
P(f) g=P_{\bar{V}} g-P_{\bar{V}} P_{\bar{X}} g+P_{\bar{X}} g,
$$

where $P_{L}$ is the orthogonal projector onto the vector space $L$. The decomposition of the projector into this three terms forms the basis of our splitting procedure (for matrix equations this has been first suggested in [22]).

We proceed by substituting $g=v \cdot \nabla_{x} f-\frac{1}{\epsilon} C(f)$ into equation $(18)$. This at once gives a three-term splitting for equation (17). More precisely, for the first order Lie splitting we have to solve the equations

$$
\begin{aligned}
& \partial_{t} f=-P_{\bar{V}}\left(v \cdot \nabla_{x} f-\frac{1}{\epsilon} C(f)\right), \\
& \partial_{t} f=P_{\bar{V}} P_{\bar{X}}\left(v \cdot \nabla_{x} f-\frac{1}{\epsilon} C(f)\right) \\
& \partial_{t} f=-P_{\bar{X}}\left(v \cdot \nabla_{x} f-\frac{1}{\epsilon} C(f)\right)
\end{aligned}
$$

one after another. In the following discussion we will consider the first order Lie splitting algorithm with step size $\tau$.

We assume that the initial value for the algorithm is given in the following form

$$
f(0, x, v)=\sum_{i j} X_{i}^{0}(x) S_{i j}^{0} V_{j}^{0}(v) .
$$


First, let us consider equation (19). Since the set $\left\{V_{j}: j=1, \ldots, r\right\}$ forms an orthonormal basis of $\bar{V}$ (for each $t$ ), we have

$$
f(t, x, v)=\sum_{j} K_{j}(t, x) V_{j}(t, v), \quad K_{j}(t, x)=\sum_{i} X_{i}(t, x) S_{i j}(t),
$$

where $K_{j}(t, x)$ is the coefficient of $V_{j}$ in the corresponding basis expansion. We duly note that $K_{j}$ is a function of $x$, but not of $v$ ). We then rewrite equation (19) as follows

$$
\begin{aligned}
& \sum_{j} \partial_{t} K_{j}(t, x) V_{j}(t, v)+\sum_{j} K_{j}(t, x) \partial_{t} V_{j}(t, v) \\
& \left.=-\sum_{j}\left(V_{j}(t, \cdot), v \mapsto v \cdot \nabla_{x} f(t, x, v)-\frac{1}{\epsilon} C(f)(x, v)\right)\right) V_{j}(t, v) .
\end{aligned}
$$

The solution of this equation is given by $V_{j}(t, v)=V_{j}(0, v)=V^{0}(v)$ and

$$
-\dot{K}_{j}(t, x)=\sum_{l} c_{j l}^{1} \cdot \nabla_{x} K_{l}(t, x)-\frac{1}{\epsilon}\left(K_{j}-c_{j}^{3}(K)(x) \rho(K)(x)\right)
$$

with

$$
c_{j l}^{1}=\int v V_{j}^{0} V_{l}^{0} \mathrm{~d} v, \quad c_{j}^{3}(K)(x)=\int V_{j} h^{\mathrm{eq}}(K) \mathrm{d} v,
$$

where we have used the decomposition $f^{\mathrm{eq}}=\rho h^{\mathrm{eq}}$. The evolution equation is obtained by equating coefficients in the basis expansion. A very useful property of the present splitting is that we have to only update the $K_{j}$, but not the $V_{j}$. We further note that $c_{j l}^{1}=\left(c_{j l}^{1 ; x_{1}}, c_{j l}^{1 ; x_{2}}\right)$ (for $d=2$ ) is a vector quantity. Also note that we use $c_{j}^{3}$ here (instead of $c_{j}^{2}$ ) to keep the notation in line with [7], where $c_{j}^{2}$ was used for the term originating from the electric field (which is not present for standard fluid flow). However, since, as is briefly discussed in the conclusion, the proposed numerical method could conceivably be generalized to magnetohydrodynamic problems, we have chosen this notation.

Equation (23) is completely posed in a $d$-dimensional (as opposed to $2 d$ dimensional) space. Thus, we proceed by integrating equation 23 with initial value

$$
K_{j}(0, x)=\sum_{i} X_{i}^{0}(x) S_{i j}^{0}
$$

until time $\tau$ to obtain $K_{j}^{1}(x)=K_{j}(\tau, x)$. However, this is not sufficient as the $K_{j}^{1}$ are not necessarily orthogonal (a requirement of our low-rank representation). Fortunately, this is easily remedied by performing a QR decomposition

$$
K_{j}^{1}(x)=\sum_{i} X_{i}^{1}(x) S_{i j}^{1}
$$

to obtain orthonormal $X_{i}^{1}$ and the matrix $S_{i j}^{1}$. Once a space discretization has been introduced, this $\mathrm{QR}$ decomposition can be simply computed by using 
an appropriate function from a software package such as LAPACK. However, from a mathematical point of view, the continuous dependence on $x$ causes no issues. For example, the modified Gram-Schmidt process works just as well in the continuous formulation considered here.

Second, we proceed in a similar way for equation 20 . In this case both $V_{j}^{0}$ and $X_{i}^{1}$ are unchanged and only $S_{i j}$ is updated. The corresponding evolution equation is given by

$$
\begin{aligned}
\dot{S}_{i j}(t) & =\left(X_{i}^{1} V_{j}^{0},(x, v) \mapsto\left(v \cdot \nabla_{x} f(t, x, v)-\frac{1}{\epsilon} C(f)(x, v)\right)\right) \\
& =\sum_{l k}\left(d_{i l}^{1} \cdot c_{j k}^{1}\right) S_{l k}+\frac{1}{\epsilon}\left(S_{i j}(t)-e_{i j}(S)\right)
\end{aligned}
$$

with

$$
d_{i l}^{1}=\int X_{i}^{1} \nabla_{x} X_{l}^{1} \mathrm{~d} x, \quad e_{i j}=\int X_{i}^{1} \rho(S) V_{j}^{0} h^{\mathrm{eq}}(S) \mathrm{d}(x, v) .
$$

Note that in this case the evolution equation depends neither on $x$ nor on $v$. We now integrate equation 24 with initial value $S_{i j}(0)=S_{i j}^{1}$ until time $\tau$ and obtain $S_{i j}^{2}=S_{i j}(\tau)$. This completes the second step of the algorithm.

Finally, we consider equation (21). Similar to the first step we have

$$
f(t, x, v)=\sum_{i} X_{i}(t, x) L_{i}(t, v), \quad L_{i}(t, v)=\sum_{j} S_{i j}(t) V_{j}(t, v) .
$$

As before, it is easy to show that the $X_{i}$ remain constant during that step. Thus, the $L_{j}$ satisfy the following evolution equation

$$
\begin{aligned}
-\dot{L}_{i}(t, v) & =\left(X_{j}^{1}, x \mapsto\left(v \cdot \nabla_{x} f(t, x, v)-\frac{1}{\epsilon} C(f)(x, v)\right)\right) \\
& =-\sum_{l}\left(d_{i l}^{1} \cdot v\right) L_{l}-\frac{1}{\epsilon}\left(L_{i}-d_{i}^{3}(L)(v)\right)
\end{aligned}
$$

with

$$
d_{i}^{3}(L)(v)=\int X_{i} \rho h^{\mathrm{eq}} d x .
$$

We then integrate equation 25 with initial value

$$
L_{i}(0, v)=\sum_{j} S_{i j}^{2} V_{j}^{0}(v)
$$

up to time $\tau$ to obtain $L_{i}^{1}(v)=L_{i}(\tau, v)$. Since, in general, the $L_{i}^{1}$ are not orthogonal we have to perform a $\mathrm{QR}$ decomposition

$$
L_{i}^{1}(v)=\sum_{j} S_{i j}^{3} V_{j}^{1}(v)
$$

to obtain $S_{i j}^{3}$ and $V_{j}^{1}$. Finally, the output of our Lie splitting algorithm is

$$
f(\tau, x, v) \approx \sum_{i j} X_{i}^{1}(x) S_{i j}^{3} V_{j}^{1}(v) .
$$


For simplicity, we have introduced the low-rank algorithm in the context of the first order Lie splitting here. However, the extension to second-order Strang splitting, which we use in the numerical simulations conducted in section 5, is straightforward.

Note that, to some extend, the algorithm introduced here has certain similarities with a lattice Boltzmann method. In particular, the $X_{i}(t, x)$ roughly correspond to the $f_{i}(t, x)$ in the introduction. However, there are important differences. In a lattice Boltzmann method the distribution function $f$ would be represented as

$$
f(t, x, v)=\sum_{i} W_{i} f_{i}(t, x) \delta\left(v-e_{i}\right) .
$$

This yields the correct moments according to equations (2) and (3). For the proposed algorithm, however, we consider the functions $V_{j}(t, v)$ which are propagated in time. Thus, we do not consider only a single velocity per $X_{i}$ but rather a distribution of velocities.

\subsection{Discretization}

The evolution equations for $S_{i j}$ and $L_{i}$ do not involve any spatial derivatives and thus require no further discretization (with the exception of the coefficients, which are constant during the corresponding sub-step).

However, the evolution equation that describe the dynamics of the $K_{j}$ are given by (for simplicity we only consider the two-dimensional case here; however, the extension to three dimensions is immediate)

$$
\partial_{t} K_{j}=-\sum_{l}\left(c_{j l}^{1 ; x_{1}} \partial_{x_{1}} K_{l}+c_{j l}^{1 ; x_{2}} \partial_{x_{2}} K_{l}\right)-\frac{1}{\epsilon}\left(K_{j}-c_{j}^{3}(K)(x) \rho(K)(x)\right) .
$$

Since this is a constant-coefficient advection, we can choose virtually any space discretization scheme (finite differences, finite volumes, etc.) to obtain

$$
\partial_{t} K=A K-\frac{1}{\epsilon}\left(K-c_{j}^{3}(K) \rho(K)\right),
$$

where $K=\left[K_{1}, \ldots, K_{r}\right]$ and $A$ is a matrix that represents the discretized differential operator.

Let us pause here for a moment. In the literature a number of different techniques have been developed to solve the Euler equations (or, more generally, fluid flow where sharp gradients occur). Often such techniques are based on upwind schemes. While implementing upwind schemes for a scalar constant coefficient advection equation is a rather simple task, the (non-scalar and nonlinear) nature of the Euler equations makes this significantly more challenging in practice. For a good review we refer the reader to 32 . One way to generalize upwind schemes is to solve a Riemann problem at the cell interface, which can incur a significant computational cost. Now, note that since equations 26) are constant-coefficient advections, most of these difficulties are avoided for the numerical scheme proposed here. Thus, upwind schemes can be implemented 
relatively easily as part of the proposed numerical algorithm. We will not explore this topic further in the present paper, but we consider this as future work.

In principle, equation (27) can be solved by an appropriate time integrator. Note, however, that using an explicit method would introduce a CFL condition. In this case we would use sub-stepping. That is, a smaller time step is used to solve (27) compared to the splitting scheme. However, this can be avoided by employing a semi-Lagrangian approach (as discussed in the following) or a spectral approach (as discussed in the next section). To do that we first apply a further splitting procedure to equation 26 . For Lie splitting this yields

$$
K(\tau, \cdot) \approx \varphi_{\tau}^{\epsilon}\left(\mathrm{e}^{-\tau c^{1 ; x_{2}} \partial_{x_{2}}} \mathrm{e}^{-\tau c^{1 ; x} \partial_{x_{1}}} K(0, \cdot)\right),
$$

where $\varphi_{\tau}^{\epsilon}$ is the partial flow generated by the collision term. The crucial part is the computation of

$$
M(t, x)=\mathrm{e}^{-\tau c^{1 ; x_{1}} \partial_{x_{1}}} M(0, x)
$$

which is equivalent to the partial differential equation

$$
\partial_{t} M(t, x)=-c^{1 ; x_{1}} \partial_{x_{1}} M(t, x) .
$$

Now, since $c^{1 ; x_{1}}$ is symmetric, there exists an orthogonal matrix $T$ such that $T c^{1 ; x_{1}} T^{T}=D$, where $D$ is a diagonal matrix. All the ingredients can be computed efficiently as $c^{1 ; x_{1}} \in \mathbb{R}^{r \times r}$ (i.e. these are small matrices). We now change variables to $\bar{M}=T M$ and obtain

$$
\partial_{t} \bar{M}_{j}(t, x)=-D_{j j} \partial_{x_{1}} \bar{M}(t, x) .
$$

This is now a set of scalar one-dimensional advection equation with constant coefficients and can thus be treated by an arbitrary semi-Lagrangian approach.

\subsection{Spectral discretization}

Pseudo-spectral methods are widely used in some fluid problems (for example, for turbulent DNS simulations [13, 34]). Here we will show that (true) spectral methods can be very naturally incorporated into the proposed low-rank scheme. To do that we perform the Fourier transformation with respect to $x$ of equation (26). This yields

$$
\partial_{t} \hat{K}(t, k)=A(k) \hat{K}(t, k)-\frac{1}{\epsilon}\left(\hat{K}-c^{3}(K) \rho(K)\right),
$$

where $\hat{K}_{j}$ denotes the Fourier transform of $K_{j}$ and we have defined $\hat{K}=$ $\left[\hat{K}_{1}, \ldots, \hat{K}_{r}\right]$ and $K=\left[K_{1}, \ldots, K_{r}\right]$. This would be sufficient for a pseudospectral approach. However, we can turn this into a spectral method by further splitting equation (28). This is possible since we only have to treat constantcoefficient advection equations and the nonlinear term (i.e. the collision operator) is free of spatial derivatives. In particular, this is in contrast to the 
Navier-Stokes equations, where the nonlinear terms involve spatial differentiation. For Lie splitting this yields

$$
\hat{K}(\tau, k) \approx \varphi_{\tau}^{\epsilon}\left(\mathrm{e}^{\tau A(k)} \hat{K}(0, k)\right),
$$

where $\varphi_{\tau}^{\epsilon}$ is the partial flow generated by the collision term. The exponential can be readily computed in Fourier space as $A(k) \in \mathbb{R}^{r \times r}$ (and thus we only have to compute the exponential of a small matrix). We also note that this approach is, obviously, not encumbered by a CFL condition.

\subsection{Computational efficiency}

In this section, we will discuss the computational characteristics of the proposed algorithm. Solving the evolution equations is at most $\mathcal{O}\left(r n^{d}\right)$ (both in terms of cost as well as in terms of storage), where $n$ is the number of grid points per direction. As we will see in section 5 it is often sufficient to use significantly fewer grid points in the velocity (i.e. $v$ ) directions than in the spatial (i.e. $x$ ) directions. Thus, the evolution equation for $K$, equation $(23)$, dominates the computational effort. This, in particular, makes the comparison to lattice Boltzmann methods (which only have to integrate $x$-dependent quantities) more favorable.

However, in addition, for the proposed numerical method we have to compute various coefficients. To compute the coefficients $c_{j l}^{1}$ and $d_{i l}^{1}$ requires a computational cost of $\mathcal{O}\left(r^{2} n^{d}\right)$ and $\mathcal{O}\left(r^{2}\right)$ storage. Now, naively computing $c_{j}^{3}$ and $d_{i}^{3}$ would be quite expensive and could easily dominate the run time of our algorithm. However, we can accomplish this with a computational cost of $\mathcal{O}\left(r^{2} n^{d}\right)$. To do that we proceed as follow. First, we write

$$
h^{\mathrm{eq}}(x, v)=\frac{1}{(2 \pi)^{d / 2}} \exp \left(-\frac{v^{2}}{2}\right) \sum_{k} h_{k}^{X}(x) h_{k}^{V}(v),
$$

where the sum is over 10/6 $(d=3 / 2)$ entries and each $h^{X}$ and $h^{V}$ is a monomial (see the expansion in equation (8)). Thus, we exploit the low-rank expansion of $h^{\mathrm{eq}}$. Then we rewrite $c_{j}^{3}$ as follows

$$
c_{j}^{3}(x)=\sum_{k} h_{k}^{X}(x) I_{j k}^{1}, \quad I_{j k}^{1}=\frac{1}{(2 \pi)^{d / 2}} \int V_{j}(v) \exp \left(-\frac{v^{2}}{2}\right) h_{k}^{V}(v) \mathrm{d} v
$$

and $d_{j}^{3}$ as follows

$$
d_{i}^{3}(v)=\frac{1}{(2 \pi)^{d / 2}} \exp \left(-\frac{v^{2}}{2}\right) \sum_{k} h_{k}^{V}(v) I_{i k}^{2}, \quad I_{i k}^{2}=\int X_{i} \rho h_{k}^{X} \mathrm{~d} x .
$$

Both computing the integrals and summing the results to obtain $c_{j}^{3}$ and $d_{i}^{3}$ requires a computational cost of $\mathcal{O}\left(r^{2} n^{d}\right)$. Finally, we can use $c_{j}^{3}$ to compute $e_{i j}$ as follows

$$
e_{i j}=\int X_{i} \rho c_{j}^{3} d x
$$


This has a computational cost of $\mathcal{O}\left(r^{2} n^{d}\right)$. Thus, the entire algorithm can be implemented with a computational cost of $\mathcal{O}\left(r^{2} n^{d}\right)$ and a storage cost of $\mathcal{O}\left(r n^{d}\right)$. In practice, computing these coefficients might even be faster as, for example, the computation of $I_{i k}^{2}$ is limited by the memory loads of $X_{i}$ and $\rho$ ( $h_{k}^{X}$ is a monomial which we can easily computed on the fly).

One might be worried that the proposed algorithm requires $\mathcal{O}\left(r^{2} n^{d}\right)$ arithmetic operations. However, we only require $\mathcal{O}\left(r n^{d}\right)$ memory operations. The latter, in a reasonable implementation, dominates the performance of the algorithm on all present and, most likely, all future computer systems. A hope is that (especially in three-dimensions) the rank $r$ can be choosen smaller than the number of PDEs in an (off-grid) lattice Boltzmann method. Then, from that perspective, the amount of memory required and the number of memory operations we have to perform is reduced. On the other hand, the number of arithmetic operations is increased. This is precisely the kind of numerical algorithm that is expected to perform very well on the next generation of supercomputers (i.e. exascale systems). Also such algorithms are desperately needed to fully exploit accelerators, such as graphic processing units and the Intel Xeon Phi. For more information we refer the reader to the ASCAC report on exascale computing [1].

\section{The numerical algorithm in the inviscid limit}

An important consideration for the present algorithm is the limit $\epsilon \rightarrow 0$. As has been outlined in section 2, the continuous problem (i.e. the Boltzmann equation) converges to the Euler equations in this case. To put this statement in the present framework, in the limit $\epsilon \rightarrow 0$ the solution of the Boltzmann equation yields a Maxwell-Boltzmann distribution in phase space.

In general, however, there is no guarantee that a numerical approximation conserves this behavior. However, in the present section we show that each part of the projector-splitting satisfies a very similar constraint.

First, we consider the evolution equations for $K_{j}$ (i.e. equation (23)). If we take $\epsilon \rightarrow 0$ we obtain the constraint

$$
K_{j}-c_{j}^{3}(K)(x) \rho(K)(x)=0
$$

which can be written as

$$
K_{j}=\frac{\rho(K)}{(2 \pi)^{d / 2}} \int V_{j}(v) \exp \left(-\frac{1}{2}(v-u(K))^{2}\right) \mathrm{d} v .
$$

This is just the projection of the Maxwell-Boltzmann distribution onto the space spanned by the $V_{j}$. Thus, as long as $\exp \left(-\frac{1}{2}(v-u(K))^{2}\right)$ can be represented accurately in the low-rank manifold (which as we have discussed in section 2 is indeed the case for weakly compressible flows) this sub-flow of the splitting algorithm respects the constraints imposed by the continuous problem. 
Now, let us consider the evolution equations for $L_{i}$ (i.e. equation (25)). For $\epsilon \rightarrow 0$ we obtain

$$
L_{i}=\int X_{i} \rho(L) \exp \left(-\frac{1}{2}(v-u(L))^{2}\right) \mathrm{d} x .
$$

This takes the Maxwell-Boltzmann distribution and projects it onto the space spanned by the $X_{i}$. Thus, we again conclude that if the corresponding lowrank manifold can accurately represent the Boltzmann-Maxwell distribution our numerical algorithm will naturally enforce the corresponding constraint.

Having considered both the evolution equations for $K_{j}$ and $L_{i}$, it should come as no surprise that we obtain a very similar result for the evolution equations for $S_{i j}$ (i.e. equation (24)). In this setting we obtain

$$
S_{i j}=\int X_{i} V_{j} \rho(S) \exp \left(-\frac{1}{2}(v-u(S))^{2}\right) \mathrm{d}(x, y)
$$

which once again is just the projection onto the space spanned by the $X_{i} V_{j}$. Thus, if we can assume that our low-rank approximation is able to exactly represent the Maxwell-Boltzmann distribution, then the dynamical low-rank splitting would yield exactly the correct form of the distribution function $f$.

\section{Numerical results}

In this section we will perform numerical simulations with the proposed algorithm. As a comparison we consider a classic fluid solver that uses the second order MacCormack method.

\subsection{Propagation of sound waves}

As the first test case we consider a simple plane wave propagating in the $y$ direction. It can be easily shown that, if we can neglect the nonlinear term in the Navier-Stokes equations (i.e. for small velocities), the damped wave equation

$$
\partial_{t t} \rho+\mu \Delta\left(\partial_{t} \rho\right)=\Delta \rho
$$

is obtained. Due to the ideal gas law $p=\rho$ this can also be written as a pressure wave. For small damping (i.e. small viscosity $\mu$ ) we obtain the plane wave solutions

$$
\rho(t, x, y)=1+\delta \sin \left(k_{x} x-\omega t\right)+\delta \sin \left(k_{y} y-\omega t\right)
$$

with $\omega$ the frequency, $\left(k_{x}, k_{y}\right)$ the wave vector, and $\delta$ the amplitude of the wave. Since the speed of sound is equal to unity, frequency and wave vector are coupled by the dispersion relation $\omega^{2}=k_{x}^{2}+k_{y}^{2}$.

For the numerical example we consider the initial value

$$
\begin{aligned}
\rho(0, x, y) & =1+\delta \sin (2 \pi y) \\
u_{1}(0, x, y) & =0 \\
u_{2}(0, x, y) & =\delta \sin (2 \pi y)
\end{aligned}
$$



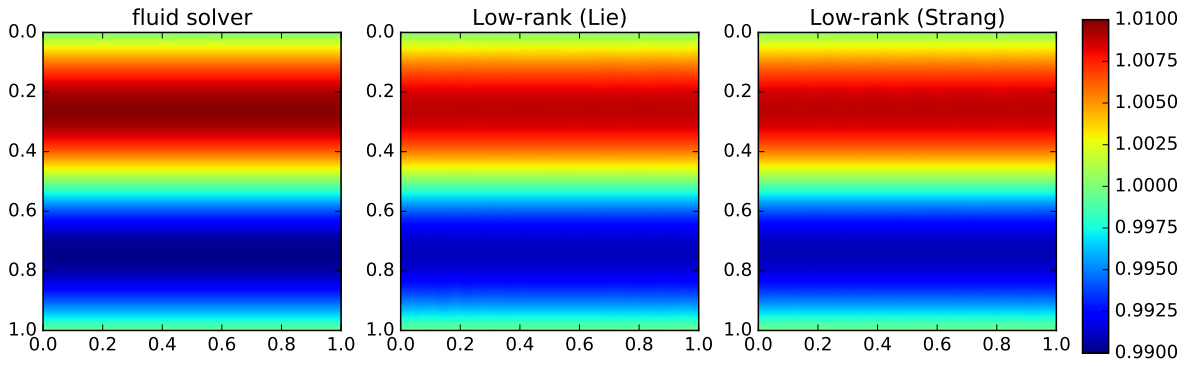

Figure 1: The density $\rho$ at time $t=1$ is shown for the classic fluid solver, Lie splitting, and Strang splitting (with $\epsilon=10^{-3}$ ). For all simulations centered differences with 128 grid points per direction are used and the rank is set to 10. The time step size for the classic fluid solver is set to $\tau=7 \cdot 10^{-3}$ (a CFL number of 0.9). For Lie splitting $\tau=0.1$ and for Strang splitting $\tau=0.2$ has been used.

on the domain $\Omega=[0,1] \times[0,1]$. As described above, for small viscosity and $\delta \ll 1$ this results in a plane wave solution traveling in the $y$-direction with unit speed. An interesting point here is that the step size of any explicit numerical method would be dictated by the CFL condition imposed by the speed of sound. That is, it would have to satisfy $\tau \leq h$, where $\tau$ is the time step size and $h$ is the grid spacing. On the other hand, the dynamic low-rank splitting proposed here should be able to take time steps dictated by accuracy (i.e. time steps that are significantly larger).

The results presented in Figure 1 are meant to check this reasoning and to verify the code in this simple setting. We observe that with the dynamical lowrank Strang splitting we can take almost 30 times larger time steps compared to the MacCormack method. The low rank approximation does not conserve mass exactly. However, in this setting the conservation of mass is still acceptable (on the order of $10^{-6}$ ), especially considering that we take quite large time steps.

\subsection{Shear flow}

Here we consider a shear flow that is given by

$$
\begin{gathered}
\rho(0, x, y)=1 \\
u_{1}(0, x, y)=v_{0} \begin{cases}\tanh \left(\frac{y-\frac{1}{4}}{\Delta}\right) & y \leq \frac{1}{2} \\
\tanh \left(\frac{\frac{3}{4}-y}{\Delta}\right) & y>\frac{1}{2}\end{cases} \\
u_{2}(0, x, y)=\delta \sin (2 \pi x) .
\end{gathered}
$$

That is, we have a velocity profile in the $y$-direction that changes relatively abruptly from $v_{0}=0.1$ to $-v_{0}$ (as we have chosen $\Delta=1 / 30$ ). A small perturbation $\left(\delta=5 \cdot 10^{-3}=0.05 \cdot v_{0}\right)$ is then added to the velocity in the $y$-direction. This problem has been used as a test problem for (mostly incompressible) flow in a number of publications [4, 19, 8]. 

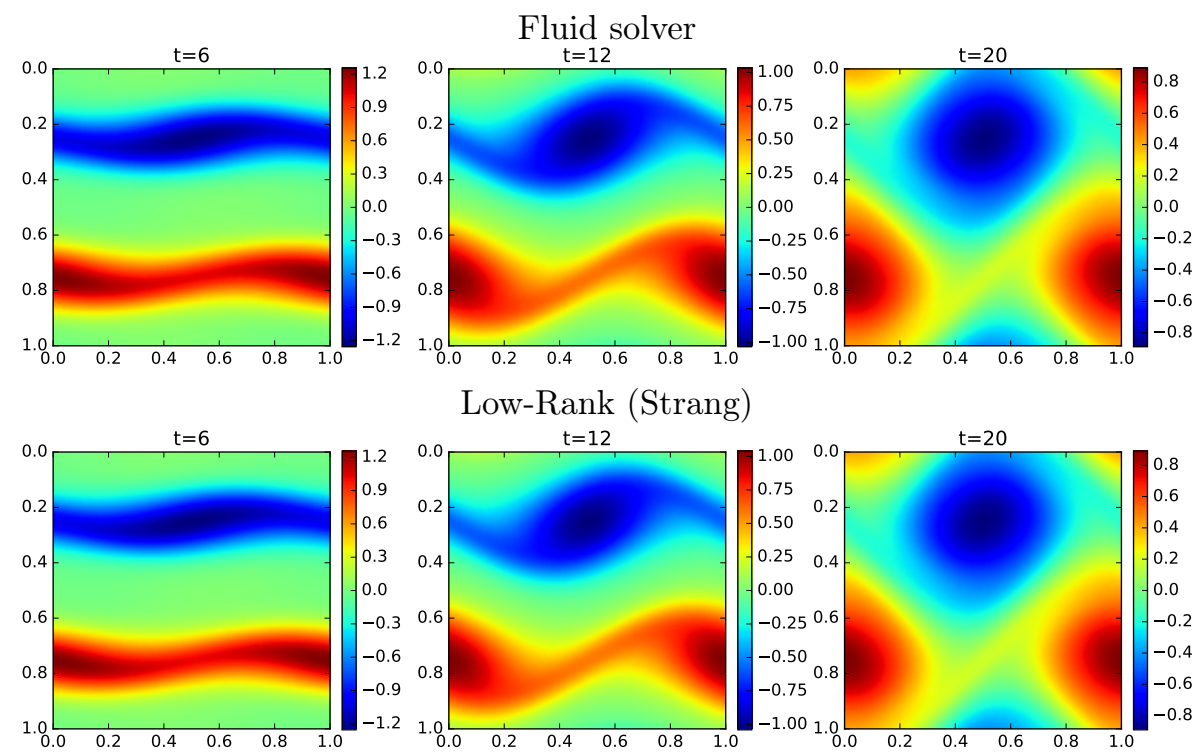

Low-Rank (Strang)

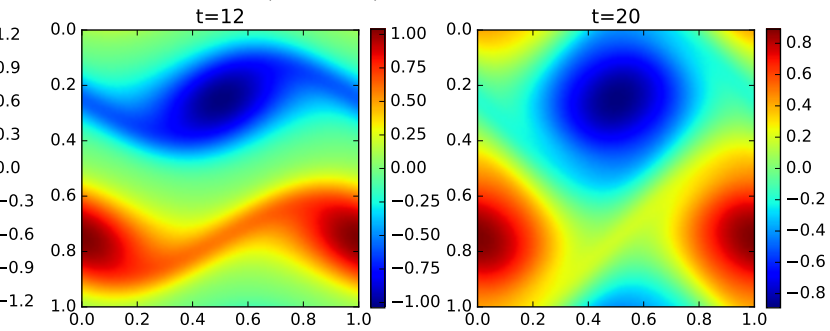

Figure 2: The time evolution of the vorticity $\omega=\partial_{x} u_{2}-\partial_{y} u_{1}$ for the shear flow given by initial values 30 is shown (with $R e=300$ ). The results from a classic fluid solver are shown on the top and the results from the dynamical low-rank splitting are shown on the bottom. For all simulations centered differences with 128 grid points per direction are used and the rank is set to 10. The time step size for the classic fluid solver is set to $\tau=7 \cdot 10^{-3}$ (a CFL number of 0.9 ), while for the low-rank solver the time step size is set to $\tau=0.1$. For the low-rank implementation Strang splitting is used.

First, we consider a modest Reynolds number $(\operatorname{Re}=300)$. The corresponding results are shown in Figure2. As is common for such studies we have plotted the vorticity. We observe excellent agreement between the proposed low-rank algorithm and the classic fluid solver. Let us also note that the low-rank algorithm is not encumbered by a CFL condition. In fact, we can take a time step that is almost 15 times as large compared to the fluid solver.

Second, we increase the Reynolds number to $R e=1000$. This is a more challenging problem in the sense that finer structures appear in the solution. The numerical results are shown in Figure 3. We once again observe excellent agreement between our low-rank algorithm and the classic fluid solver. In fact, all of the conclusions drawn for the case $\operatorname{Re}=300$ can be applied to the present case as well.

The last point we want to make here is that it is usually not necessary to use a large number of grid points in the velocity direction. To demonstrate this, we have repeated our numerical experiment with only 16 grid points in the $v$-directions, while still using 128 grid points in the space directions. In that setting the computational performance is completely dictated by solving equation (23). Nevertheless, as Figure 4 demonstrates, the numerical results show excellent agreement compared to Figure 3, where 128 grid points where used in the velocity directions. 

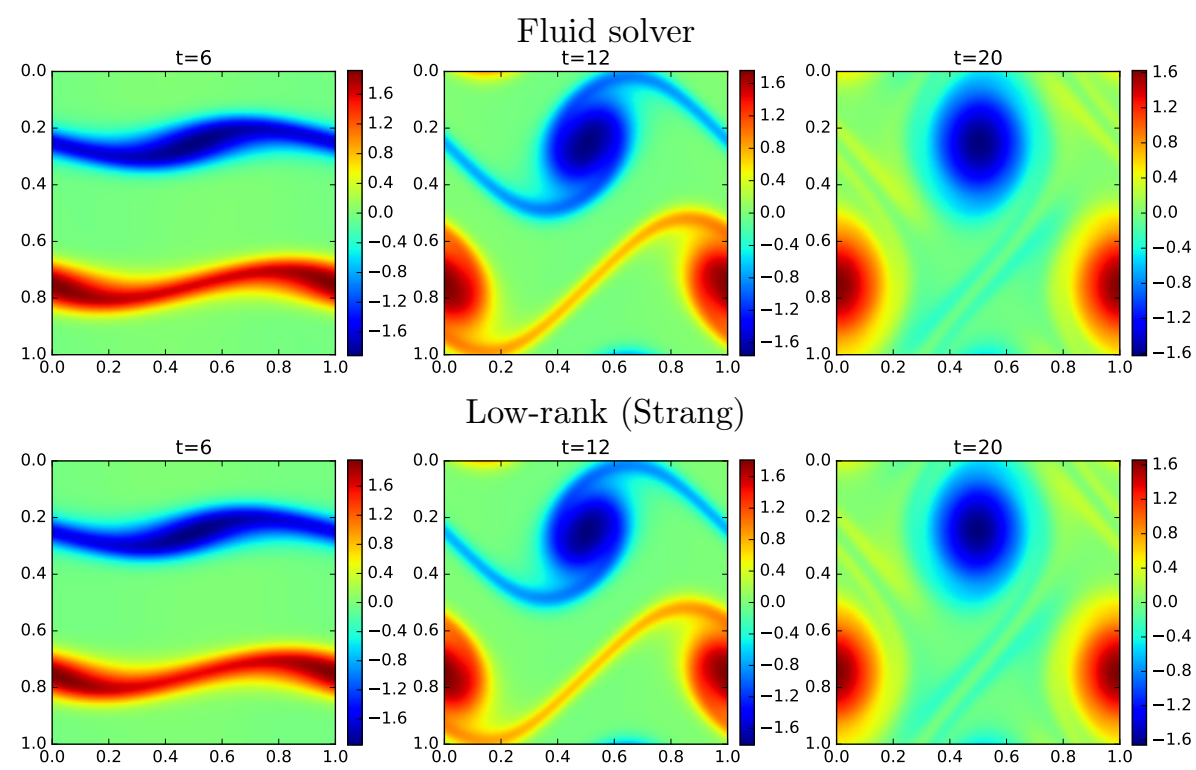

Figure 3: The time evolution of the vorticity $\omega=\partial_{x} u_{2}-\partial_{y} u_{1}$ for the shear flow given by initial values 30 is shown (with $\operatorname{Re}=1000$ ). The results from a classic fluid solver are shown on the top and the results from the dynamical low-rank splitting are shown on the bottom. For all simulations centered differences with 128 grid points per direction are used and the rank is set to 10 . The time step size for the classic fluid solver is set to $\tau=7 \cdot 10^{-3}$ (a CFL number of 0.9), while for the low-rank solver it is set to $\tau=0.1$. For the low-rank implementation Strang splitting is used.

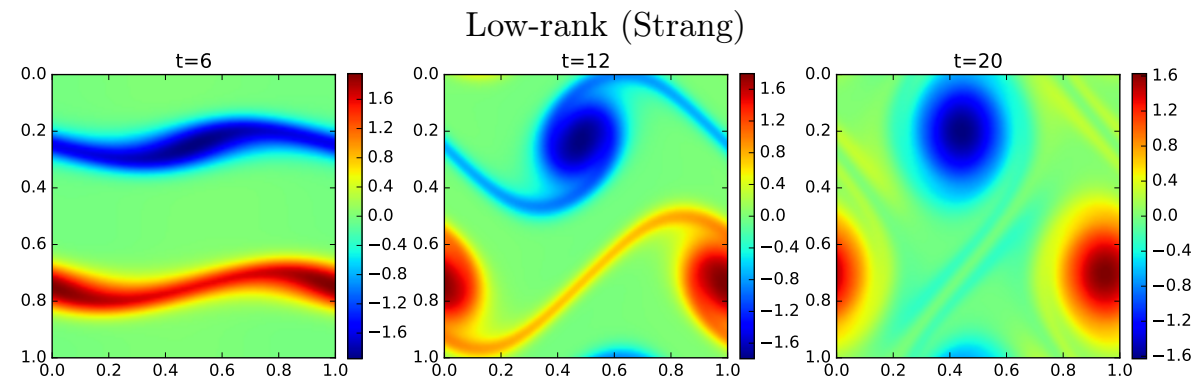

Figure 4: The time evolution of the vorticity $\omega=\partial_{x} u_{2}-\partial_{y} u_{1}$ for the shear flow given by initial values 30 is shown (with $R e=1000$ ). The dynamical low-rank splitting is used as the integrator. For all simulations centered differences with 128 grid points in the space directions and 16 grid points in the velocity directions are used. The time step size is set to $\tau=0.1$ and Strang splitting has been employed. 


\section{Conclusion \& Outlook}

We have introduced a numerical algorithm for solving the weakly compressible Navier-Stokes equations that is based on a dynamical low-rank splitting algorithm. The behavior of this algorithm has been investigated and numerical simulations have been conducted that show excellent agreement with a classic fluid solver.

The algorithm has been considered in the context of weakly compressible isothermal flow with periodic boundary conditions. However, this restrictions are not fundamental problems. For example, the extension to temperature dependent flows is immediate. In fact, only a time and space dependent $\theta$ has to be introduced in section 2 This (slightly) changes the collision operator, but the numerical method remains virtually unaffected. The expansion (8) is only valid for small velocities (i.e. weakly compressible flow). However, this does not mean that we can not efficiently represent the solution by a low-rank function. In fact, it is not even clear that equation (8) is the best low-rank approximation (i.e. the approximation with the smallest rank) one can obtain. We have only considered periodic boundary conditions here. However, similar to the lattice Boltzmann method, no-slip boundary conditions can be imposed by a 'bounce-back' scheme. All of this is the subject of future research.

Furthermore, the method proposed here offers a path forward for simulations that need to resolve some kinetic effects. Such problems are common in various fields of plasma physics. Full scale simulations with the Boltzmann (collisional Vlasov) equation are often prohibitive from a computational point of view. However, as has been shown in [7] low-rank approximations are still able to resolve a range of kinetic effects quite well. The method proposed here would thus conceivably allow us to extend fluid models (say magnetohydrodynamics) to a regime in which kinetic effects are needed.

\section{Acknowledgments}

We would like to thank Christian Lubich (University of Tübingen) for the many helpful discussions.

\section{References}

[1] S. Ashby et al. The opportunities and challenges of exascale computing. Report of the ASCAC Subcommittee on Exascale Computing, 2010.

[2] C. Bardos, F. Golse, and C.D. Levermore. Fluid dynamic limits of kinetic equations II. Convergence proofs for the Boltzmann equation. Commun. Pure Appl. Math, 46(5):667-753, 1993.

[3] C. Bardos, F. Golse, and D. Levermore. Fluid dynamic limits of kinetic equations. I. Formal derivations. J. Stat. Phys, 63(1-2):323-344, 1991. 
[4] J.B. Bell, P. Colella, and H.M Glaz. A second-order projection method for the incompressible Navier-Stokes equations. J. Comput. Phys., 85(2):257$283,1989$.

[5] S. Chen and G.D. Doolen. Lattice Boltzmann method for fluid flows. Annu. Rev. Fluid Mech., 30(1):329-364, 1998.

[6] D. Conte and C. Lubich. An error analysis of the multi-configuration timedependent Hartree method of quantum dynamics. ESAIM Math. Model. Numer. Anal., 44(4):759-780, 2010.

[7] L. Einkemmer and C. Lubich. A low-rank projector-splitting integrator for the Vlasov-Poisson equation. preprint, arXiv:1801.01103, 2018.

[8] L. Einkemmer and M. Wiesenberger. A conservative discontinuous Galerkin scheme for the 2D incompressible Navier-Stokes equations. Comput. Phys. Commun., 185(11):2865-2873, 2014.

[9] A. Fakhari and T. Lee. Numerics of the lattice Boltzmann method on nonuniform grids: standard LBM and finite-difference LBM. Comput. Fluids, 107:205-213, 2015.

[10] J. Haegeman, C. Lubich, I. Oseledets, B. Vandereycken, and F. Verstraete. Unifying time evolution and optimization with matrix product states. Phys. Rev. B, 94(16):165116, 2016.

[11] T. Jahnke and W. Huisinga. A dynamical low-rank approach to the chemical master equation. J. Math. Biol., 70(8):2283-2302, 2008.

[12] E. Kieri, C. Lubich, and H. Walach. Discretized dynamical low-rank approximation in the presence of small singular values. SIAM J. Numer. Anal., 54(2):1020-1038, 2016.

[13] J. Kim, P. Moin, and R. Moser. Turbulence statistics in fully developed channel flow at low Reynolds number. J. Fluid Mech., 177:133-166, 1987.

[14] O. Koch and C. Lubich. Dynamical low-rank approximation. SIAM J. Matrix Anal. Appl., 29(2):434-454, 2007.

[15] O. Koch and C. Lubich. Dynamical tensor approximation. SIAM J. Matrix Anal. Appl., 31(5):2360-2375, 2010.

[16] K. Kormann. A semi-Lagrangian Vlasov solver in tensor train format. SIAM J. Sci. Comput., 37(4):613-632, 2015.

[17] A. Krämer, K. Küllmer, D. Reith, W. Joppich, and H. Foysi. SemiLagrangian off-lattice Boltzmann method for weakly compressible flows. Phys. Rev. E, 95:023305, 2017. 
[18] P. Lallemand and L. Luo. Theory of the lattice Boltzmann method: Dispersion, dissipation, isotropy, Galilean invariance, and stability. Phys. Rev. E, 61:6546-6562, 2000.

[19] J. Liu and C. Shu. A high-order discontinuous Galerkin method for 2D incompressible flows. J. Comput. Phys., 160(2):577-596, 2000.

[20] C. Lubich. From quantum to classical molecular dynamics: reduced models and numerical analysis. European Mathematical Society, 2008.

[21] C. Lubich. Time integration in the multiconfiguration time-dependent Hartree method of molecular quantum dynamics. Appl. Math. Res. Express, 2015(2):311-328, 2015.

[22] C. Lubich and I.V. Oseledets. A projector-splitting integrator for dynamical low-rank approximation. BIT Numer. Math., 54(1):171-188, 2014.

[23] C. Lubich, I.V. Oseledets, and B. Vandereycken. Time integration of tensor trains. SIAM J. Numer. Anal., 53(2):917-941, 2015.

[24] C. Lubich, T. Rohwedder, R. Schneider, and B. Vandereycken. Dynamical approximation by hierarchical Tucker and tensor-train tensors. SIAM J. Matrix Anal. Appl., 34(2):470-494, 2013.

[25] C. Lubich, B. Vandereycken, and H. Walach. Time integration of rankconstrained Tucker tensors. Preprint, arXiv:1709.02594, 2017.

[26] H. Mena, A. Ostermann, L. Pfurtscheller, and C. Piazzola. Numerical lowrank approximation of matrix differential equations. arXiv:1705.10175, 2017.

[27] H.-D. Meyer, F. Gatti, and G. A. Worth. Multidimensional quantum dynamics. John Wiley \& Sons, 2009.

[28] H.D. Meyer, U. Manthe, and L. S. Cederbaum. The multi-configurational time-dependent Hartree approach. Chem. Phys. Letters, 165(1):73-78, 1990.

[29] M. Min and T. Lee. A spectral-element discontinuous Galerkin lattice Boltzmann method for nearly incompressible flows. J. Comput. Phys., 230(1):245-259, 2011.

[30] E. Musharbash, F. Nobile, and T. Zhou. Error analysis of the Dynamically Orthogonal approximation of time dependent random PDEs. SIAM J. Sci. Comput., 37(2):A776-A810, 2015.

[31] A. Nonnenmacher and C. Lubich. Dynamical low-rank approximation: applications and numerical experiments. Math. Comput. Simul., 79(4):13461357, 2008. 
[32] B. Van Leer. Upwind and high-resolution methods for compressible flow: From donor cell to residual-distribution schemes. In 16th AIAA Computational Fluid Dynamics Conference, page 3559, 2006.

[33] K. Xu and X. He. Lattice Boltzmann method and gas-kinetic BGK scheme in the low-Mach number viscous flow simulations. J. Comput. Phys., 190(1):100-117, 2003.

[34] M. Yokokawa, K. Itakura, A. Uno, T. Ishihara, and Y. Kaneda. 16.4-Tflops direct numerical simulation of turbulence by a Fourier spectral method on the Earth Simulator. In Proceedings of the 2002 ACM/IEEE conference on Supercomputing, pages 1-17, 2002. 